\title{
A new Calculation Procedure for Spatial Impulse Responses in Ultrasound
}

\author{
Jensen, Jørgen Arendt
}

Published in:

Acoustical Society of America. Journal

Link to article, DOI:

$10.1121 / 1.424654$

Publication date:

1999

Document Version

Publisher's PDF, also known as Version of record

Link back to DTU Orbit

Citation (APA):

Jensen, J. A. (1999). A new Calculation Procedure for Spatial Impulse Responses in Ultrasound. Acoustical Society of America. Journal, 105(6), 3266-3274. https://doi.org/10.1121/1.424654

\section{General rights}

Copyright and moral rights for the publications made accessible in the public portal are retained by the authors and/or other copyright owners and it is a condition of accessing publications that users recognise and abide by the legal requirements associated with these rights.

- Users may download and print one copy of any publication from the public portal for the purpose of private study or research.

- You may not further distribute the material or use it for any profit-making activity or commercial gain

- You may freely distribute the URL identifying the publication in the public portal

If you believe that this document breaches copyright please contact us providing details, and we will remove access to the work immediately and investigate your claim 


\title{
A new calculation procedure for spatial impulse responses in ultrasound
}

\author{
Jørgen Arendt Jensen \\ Department of Information Technology, Building 344, Technical University of Denmark, DK-2800 Lyngby, \\ Denmark
}

(Received 24 October 1997; revised 18 February 1999; accepted 25 February 1999)

\begin{abstract}
A new procedure for the calculation of spatial impulse responses for linear sound fields is introduced. This calculation procedure uses the well known technique of calculating the spatial impulse response from the intersection of a circle emanating from the projected spherical wave with the boundary of the emitting aperture. This general result holds for all aperture boundaries for a flat transducer surface, and is used in the procedure to yield the response for all types of flat transducers. An arbitrary apodization function over the aperture can be incorporated through a simple one-dimensional integration. The case of a soft baffle mounting of the aperture is also included. Specific solutions for transducer boundaries made from lines are given, so that any polygon transducer can be handled. Specific solutions for circles are also given. Finally, a solution for a general boundary is stated, and all these boundary elements can be combined to, e.g., handle annular arrays or semi-circle transducers. Results from an implementation of the approach are given and compared to previously developed solutions for a simple aperture, a complex aperture, and a Gaussian apodized circular transducer. (C) 1999 Acoustical Society of America. [S0001-4966(99)01406-X]
\end{abstract}

PACS numbers: 43.35.Cg, 43.20.Px [HEB]

\section{INTRODUCTION}

The calculation of linear, acoustic fields is most often based on the spatial impulse response approach as suggested by Tupholme and Stepanishen. ${ }^{1-3}$ Here the pulsed pressure field is found from a convolution between the acceleration of the transducer surface and the spatial impulse response. The impulse response has been found for a number of geometries (round flat piston, ${ }^{2}$ round concave, ${ }^{4,5}$ flat rectangle, ${ }^{6,7}$ and flat triangle ${ }^{8}$ ). The solutions arrived at are often complicated, since it involves the evaluation of the Rayleigh surface integral. The response depends on the relative position of the field point and many special cases exist, which makes both the derivation of the solution difficult and the evaluation of the responses cumbersome. For example, to evaluate the response from a rectangle, four synthetic rectangles are introduced, and when evaluating a triangle, three synthetic triangles are introduced to account for the 15 different possible cases of triangle shape and field point positions. This makes it necessary to use computers for evaluating and interpreting the responses, since the formulas do not readily give a useful perception of the solution.

It would be appropriate to arrive at general solutions for any geometry that would be both easy to derive analytically and fast to evaluate with a computer. This has previously been achieved by dividing the aperture surface into smaller elements like rectangles ${ }^{9}$ or triangles, ${ }^{10}$ and then summing the response for the sub-elements. Often the transducer must be divided into many elements and only a piecewise approximation to the apodization is obtained. The fitting to the actual surface is also only approximative for round or oval surfaces; even when using a triangular shape.
Spatial impulse responses from bounded and nonapodized apertures always have discontinuities due to their sharp edges, which makes it difficult to keep the full energy and spectral content in a sampled evaluation. Various techniques have been applied for coping with the discontinuities in the spatial impulse response. This has included using very high sampling frequencies, making a time adapted evaluation, or using the integrated response. Computer evaluation is, thus, always necessary, when evaluating spatial impulse responses.

This paper therefore suggests a new procedure for calculating the spatial impulse response in which the computer is involved at an earlier stage in the evaluation of the responses. The response is determined by the crossings of the boundary of the aperture by the spherical wave emitted from the field point. For flat apertures this observation makes it possible to derive a general approach for calculating the spatial impulse response for any aperture geometry and find the response with no approximation. The paper derives impulse responses for apertures described by bounding lines and circles and outlines how the response can be evaluated for apertures bounded by any polynomial in the plane's coordinates $(x, y)$. Some of the intersections need not always be calculated, and this is used to devise an optimized algorithm that only needs to find the minimum number of intersections. It is also shown in Sec. III how an arbitrary apodization can be introduced through a previously developed simple onedimensional integration, and how the solution also can be applied to both the soft baffle and rigid baffle situations. A number of examples from use of the approach are given in Sec. VII. Among these is a comparison between the tradi- 


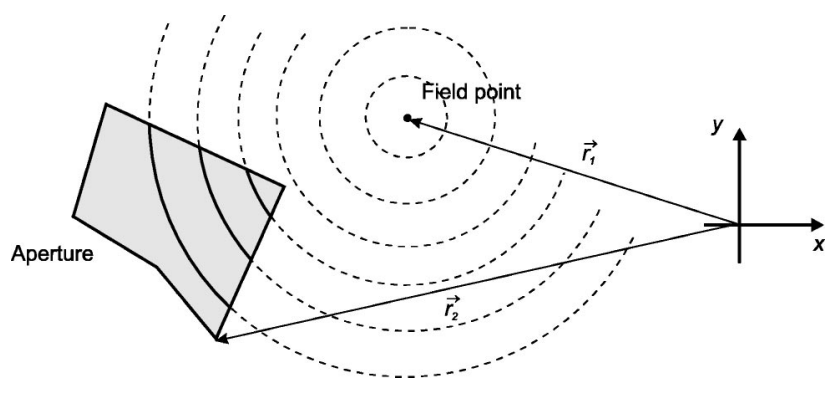

FIG. 1. Intersection of spherical waves from the field point by the aperture, when the field point is projected onto the plane of the aperture.

tional solution for a rectangle and the new approach, which both yield the same response.

\section{BASIC THEORY}

A short review of the calculation of spatial impulse responses is given in this section to facilitate the development of the new calculation procedure.

The spatial impulse response is found from the Rayleigh integral given by: $:^{2,11}$

$$
h\left(\mathbf{r}_{1}, t\right)=\int_{S} \frac{\delta\left(t-\frac{\left|\mathbf{r}_{1}-\mathbf{r}_{2}\right|}{c}\right)}{2 \pi\left|\mathbf{r}_{1}-\mathbf{r}_{2}\right|} d S
$$

when the aperture $S$ is mounted in an infinite, rigid baffle. Here $\mathbf{r}_{1}$ denotes the position of the field point, $\mathbf{r}_{2}$ denotes a position on the aperture, $c$ is the speed of sound, and $t$ is time. The integral is essentially a statement of Huyghen's principle that the field is found by summing the radiated spherical waves from all parts of the aperture. This can also be reformulated, due to acoustic reciprocity, as finding the part of the spherical wave emanating from the field point that intersects the aperture. The task is, thus, to project the field point onto the plane coinciding with the aperture, and then find the intersection of the projected spherical wave (the circle) with the active aperture as shown in Fig. 1.

Rewriting the integral into polar coordinates gives:

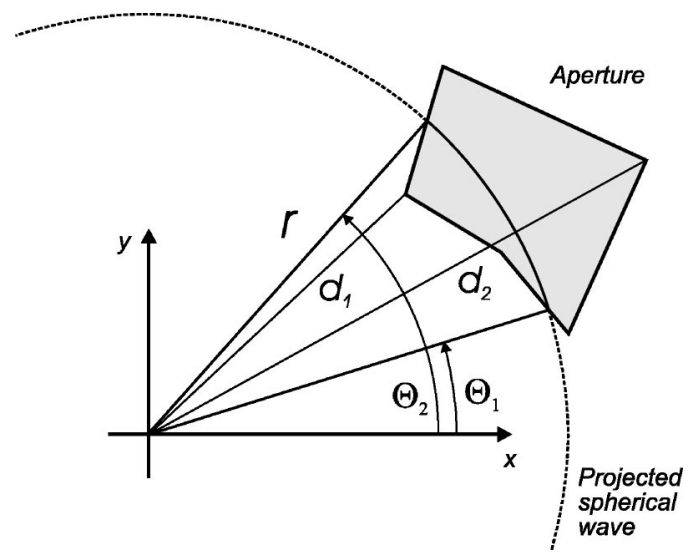

FIG. 2. Definition of distances and angles in the aperture plan for evaluating the Rayleigh integral.

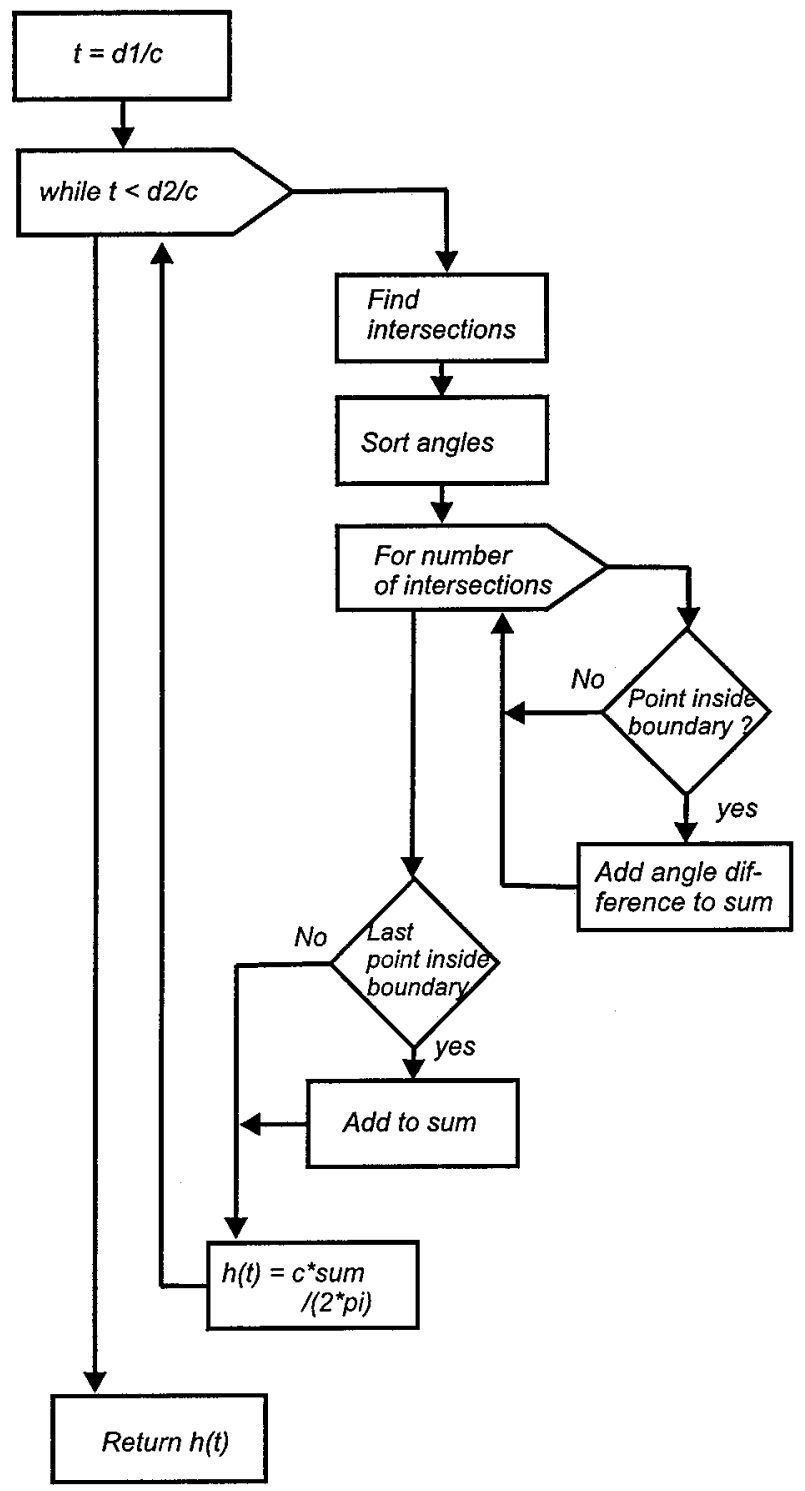

FIG. 3. Flow chart for the simple approach for calculating the spatial impulse response.

$$
h\left(\mathbf{r}_{1}, t\right)=\int_{\Theta_{1}}^{\Theta_{2}} \int_{d_{1}}^{d_{2}} \frac{\delta\left(t-\frac{R}{c}\right)}{2 \pi R} r d r d \Theta,
$$

where $r$ is the radius of the projected circle and $R$ is the distance from the field point to the aperture given by $R^{2}$ $=r^{2}+z_{p}^{2}$. Here $z_{p}$ is the field point height above the $x-y$ plane of the aperture. The projected distances $d_{1}, d_{2}$ are determined by the aperture and are the distance closest to and furthest away from the aperture, and $\Theta_{1}, \Theta_{2}$ are the corresponding angles for a given time (see Fig. 2).

Introducing the substitution $2 R d R=2 r d r$ gives

$$
h\left(\mathbf{r}_{1}, t\right)=\frac{1}{2 \pi} \int_{\Theta_{1}}^{\Theta_{2}} \int_{R_{1}}^{R_{2}} \delta\left(t-\frac{R}{c}\right) d R d \Theta .
$$

The variables $R_{1}$ and $R_{2}$ denote the edges closest to and furthest away from the field point. Finally using the substitution $t^{\prime}=R / c$ gives 


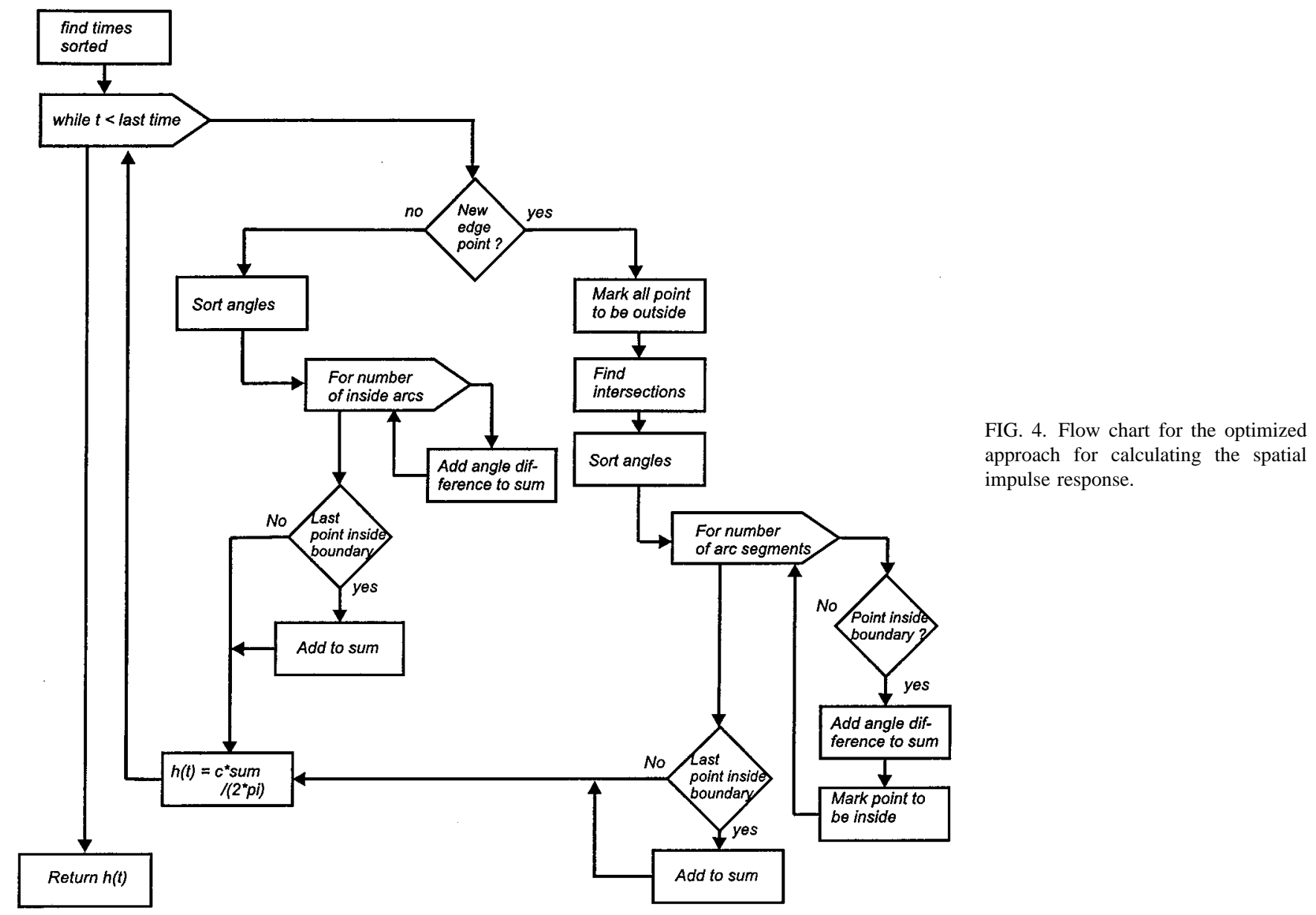

$$
h\left(\mathbf{r}_{1}, t\right)=\frac{c}{2 \pi} \int_{\Theta_{1}}^{\Theta_{2}} \int_{t_{1}}^{t_{2}} \delta\left(t-t^{\prime}\right) d t^{\prime} d \Theta .
$$

For a given time instance the contribution along the arc is constant and the integral gives

$$
h\left(\mathbf{r}_{1}, t\right)=\frac{\Theta_{2}-\Theta_{1}}{2 \pi} c
$$

when assuming the circle arc is only intersected once by the aperture. The angles $\Theta_{1}$ and $\Theta_{2}$ are determined by the intersection of the aperture and the projected spherical wave, and the spatial impulse response is, thus, solely determined by these intersections, when no apodization of the aperture is used. The response can therefore be evaluated by keeping track of the intersections as a function of time.

\section{A NEW CALCULATION PROCEDURE}

From the derivation in the last section it can be seen that the spatial impulse response in general can be expressed as

$$
h\left(\mathbf{r}_{1}, t\right)=\frac{c}{2 \pi} \sum_{i=1}^{N(t)}\left[\Theta_{2}^{(i)}(t)-\Theta_{1}^{(i)}(t)\right],
$$

where $N(t)$ is the number of arc segments that crosses the boundary of the aperture for a given time and $\Theta_{2}^{(i)}(t)$, $\Theta_{1}^{(i)}(t)$ are the associated angles of the arc. This was also noted by Stepanishen. ${ }^{12}$ The calculation can, thus, be formu- lated as finding the angles of the aperture edge's intersections with the projected spherical wave, sorting the angles, and then summing the arc angles that belong to the aperture. Finding the intersections can be done from the description of the edges of the aperture. A triangle can be described by three lines, a rectangle by four, and the intersections are then found from the intersections of the circle with the lines. This makes it possible to devise a general procedure for calculating spatial impulse responses for any flat, bounded aperture, since the task is just to find the intersections of the boundary with the circle.

The spatial impulse response is calculated from the time the aperture first is intersected by a spherical wave to the time for the intersection furthest away. The intersections are found for every time instance and the corresponding angles are sorted. The angles lie in the interval from 0 to $2 \pi$. It is then found whether the arc between two angles belongs to the aperture, and the angle difference is added to the sum, if the arc segment is inside the aperture. This yields the spatial impulse response according to Eq. (6). The approach can be described by the flow chart shown in Fig. 3.

The only part of the algorithm specific to the aperture is the determination of the intersections and the whether the point is inside the aperture. Section IV shows how this is done for polygons, Sec. V for circles, and Sec. VI for higherorder parametric boundaries.

All the intersections need not be found for all times. New intersections are only introduced, when a new edge or 


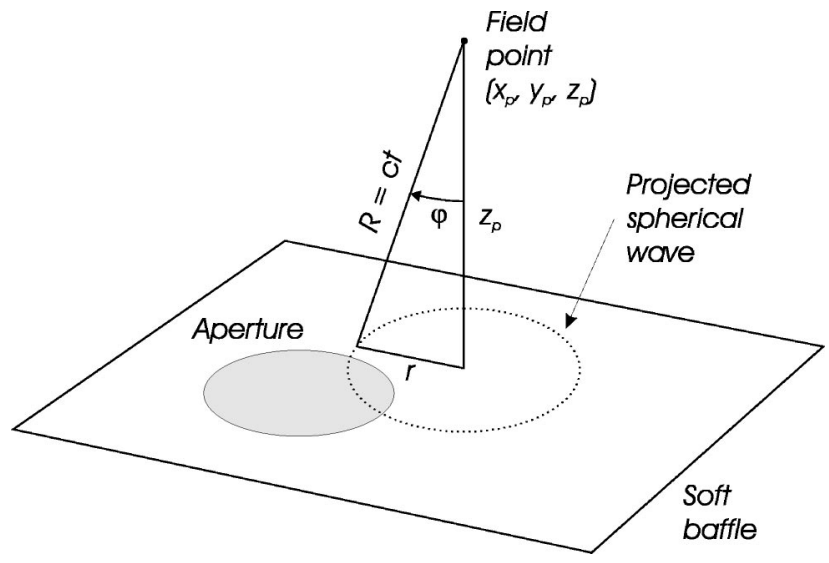

FIG. 5. Definition of angle used for a soft baffle.

corner of the aperture is met. Between times when two such corners or edges are encountered the number of intersections remains constant and only intersections, which belong to points inside the aperture need be found. Note that an aperture edge gives rise to a discontinuity in the spatial impulse response. Also testing whether the point is inside the aperture is often superfluous, since this only needs to be found once after each discontinuity in the response. These two observations can significantly reduce the number of calculations, since only the intersections affecting the response are found. The flow chart for the optimized approach is shown in Fig. 4.

The procedure first finds the number of discontinuities. Then only intersection influencing the response are calculated between two discontinuity points. This can potentially make the approach faster than the traditional approach, where the response from a number of different rectangles or triangles must be calculated.

\section{APODIZATION AND SOFT BAFFLE}

Often ultrasound transducers do not vibrate as a piston over the aperture. This can be due to the clamping of the active surface at its edges, or intentionally to reduce side lobes in the field. Applying for example a Gaussian apodization will significantly lower side lobes and generate a field with a more uniform point spread function as a function of depth. Apodization has previously been found and is introduced in Eq. (2) by writing ${ }^{13}$

$$
h\left(\mathbf{r}_{1}, t\right)=\int_{\Theta_{1}}^{\Theta_{2}} \int_{d_{1}}^{d_{2}} a_{p}(r, \Theta) \frac{\delta\left(t-\frac{R}{c}\right)}{2 \pi R} r d r d \Theta
$$

in which $a_{p}(r, \Theta)$ is the apodization over the aperture. Using the same substitutions as before yields

$$
h\left(\mathbf{r}_{1}, t\right)=\frac{c}{2 \pi} \int_{\Theta_{1}}^{\Theta_{2}} \int_{t_{1}}^{t_{2}} a_{p 1}\left(t^{\prime}, \Theta\right) \delta\left(t-t^{\prime}\right) d t^{\prime} d \Theta,
$$

where $a_{p 1}\left(t^{\prime}, \Theta\right)=a_{p}\left(\sqrt{\left(c t^{\prime}\right)^{2}-z_{p}^{2}}, \Theta\right)$. The inner integral is a convolution of the apodization function with a $\delta$-function and readily yields

$$
h\left(\mathbf{r}_{1}, t\right)=\frac{c}{2 \pi} \int_{\Theta_{1}}^{\Theta_{2}} a_{p 1}(t, \Theta) d \Theta
$$

as noted by several authors. ${ }^{13-15}$ The response for a given time point can, thus, be found by integrating the apodization function along the fixed arc with a radius of $r=\sqrt{(c t)^{2}-z_{p}^{2}}$ for the angles for the active aperture. Any apodization function can therefore be incorporated into the calculation by employing numerical integration.

Often the assumption of an infinite rigid baffle for the transducer mounting is not appropriate and another form of the Rayleigh integral must be used. For a soft baffle, in which the pressure on the baffle surface is zero, the Rayleigh-Sommerfeld integral is used. This is (Ref. 16, pp. 46-50)

$$
h_{s}\left(\mathbf{r}_{1}, t\right)=\int_{S} \frac{\delta\left(t-\frac{\left|\mathbf{r}_{1}-\mathbf{r}_{2}\right|}{c}\right)}{2 \pi\left|\mathbf{r}_{1}-\mathbf{r}_{2}\right|} \cos \varphi d S,
$$

assuming that $\left|\mathbf{r}_{1}-\mathbf{r}_{2}\right| \gg \lambda$. Here $\cos \varphi$ is the angle between the line through the field point orthogonal to the aperture plane and the radius of the spherical wave as shown in Fig. 5. The angles $\varphi$ is fixed for a given radius of the projected spherical wave and thus for a given time. It is given by

$$
\cos \varphi=\frac{z_{p}}{R}=\frac{z_{p}}{c t} .
$$

Using the substitutions from Sec. I the RayleighSommerfeld integral can then be rewritten as

$$
h_{s}\left(\mathbf{r}_{1}, t\right)=\frac{z_{p}}{2 \pi} c\left(\Theta_{2}-\Theta_{1}\right) \int_{t_{1}}^{t_{2}} \frac{\delta\left(t-t^{\prime}\right)}{c t^{\prime}} d t^{\prime} .
$$

Using the property of the $\delta$-function that

$$
\int_{-\infty}^{+\infty} g\left(t^{\prime}\right) \delta\left(t-t^{\prime}\right) d t^{\prime}=g(t)
$$

then gives

$$
h_{s}\left(\mathbf{r}_{1}, t\right)=\frac{z_{p}}{c t} \frac{\Theta_{2}-\Theta_{1}}{2 \pi} c=\frac{z_{p}}{c t} h\left(\mathbf{r}_{1}, t\right) .
$$

The spatial impulse response can, thus, be found from the spatial impulse response for the rigid baffle case by multiplying with $z_{p} /(c t)$.

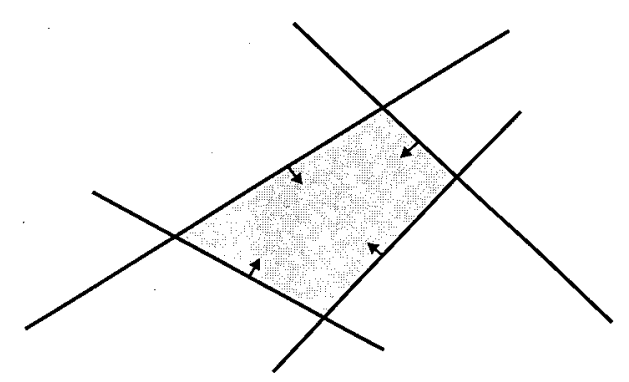

FIG. 6. Definition of bounding lines for polygon transducer. The arrows indicates the half-planes for the active aperture. 


\section{SOLUTION FOR POLYGONS}

The boundary of any polygon can be defined by a set of bounding lines as shown in Fig. 6 . The active aperture is then defined as lying on one side of the line as indicated by the arrows, and a point on the aperture must be placed correctly in relation to all lines. The test whether a point is on the aperture is thus to go through all lines and test whether the point lies in the active half space for the line, and stop if it is not. The point is inside the aperture, if it passes the test for all the lines.

The intersections are found from the individual intersections between the projected circle and the lines. They are determined from the equations for the projected spherical wave and the line:

$$
\begin{aligned}
& r^{2}=\left(x-x_{0}\right)^{2}+\left(y-y_{0}\right)^{2}, \\
& y=\alpha x+y_{1}, \\
& r^{2}=(c t)^{2}-z_{p}^{2} .
\end{aligned}
$$

Here $\left(x_{0}, y_{0}\right)$ is the center of the circle, $\alpha$ the slope of the line, and $y_{1}$ its intersect with the $y$-axis. The intersections are given from the solutions to:

$$
\begin{aligned}
0= & \left(1+\alpha^{2}\right) x^{2}+\left(2 \alpha y_{1}-2 x_{0}-2 y_{0} \alpha\right) x \\
& +\left(y_{0}^{2}+y_{1}^{2}+x_{0}^{2}-2 y_{0} y_{1}-r^{2}\right) \\
= & A x^{2}+B x+C, \\
D= & B^{2}-4 A C .
\end{aligned}
$$

The angles are

$$
\Theta=\arctan \left(\frac{y-y_{0}}{x-x_{0}}\right)
$$

Intersections between the line and the circle are only found if $D>0$. A determinant $D<0$ indicates that the circle did not intersect the line. If the line has infinite slope, the solution is found from the equation:

$$
\begin{aligned}
x & =x_{1}, \\
0 & =y^{2}-2 y_{0} y+y_{0}^{2}+\left(x_{1}-x_{0}\right)^{2}-r^{2} \\
& =A_{\infty} y^{2}+B_{\infty} y+C_{\infty},
\end{aligned}
$$

in which $A_{\infty}, B_{\infty}, C_{\infty}$ replaces $A, B, C$, respectively, and the solutions are found for $y$ rather than $x$. Here $x_{1}$ is the line's intersection with the $x$-axis.

The times for discontinuities in the spatial impulse response are given by the intersections of the lines that define the aperture's edges and by the minimum distance from the projected field point to the lines. The minimum distance is found from a line passing through the field point that is orthogonal to the bounding line. The intersection between the orthogonal line and the bounding line is:

$$
\begin{aligned}
& x=\frac{\alpha y_{p}+x_{p}-\alpha y_{1}}{\alpha^{2}+1}, \\
& y=\alpha x+y_{1},
\end{aligned}
$$

where $\left(x_{p}, y_{p}, z_{p}\right)$ is the position of the field point. For an infinite slope line the solution is $x=x_{1}$ and $y=y_{p}$. The corresponding time is:

$$
t_{i}=\frac{\sqrt{\left(x-x_{p}\right)^{2}+\left(y-y_{p}\right)^{2}+z_{p}^{2}}}{c} .
$$

The intersections of the lines are also found, and the corresponding times are calculated by Eq. (20) and sorted in ascending order. They indicate the start and end time for the response and the time points for discontinuities in the response.

\section{SOLUTION FOR CIRCULAR SURFACES}

The other basic shape for a transducer apart from rectangular shapes is the flat, round surface used for single element piston transducers and annular arrays. For these the intersections are determined by two circles as depicted in Fig. 7. Here $O_{1}$ is the center of the aperture with radius $r_{a}$

\section{Field point outside the aperture}
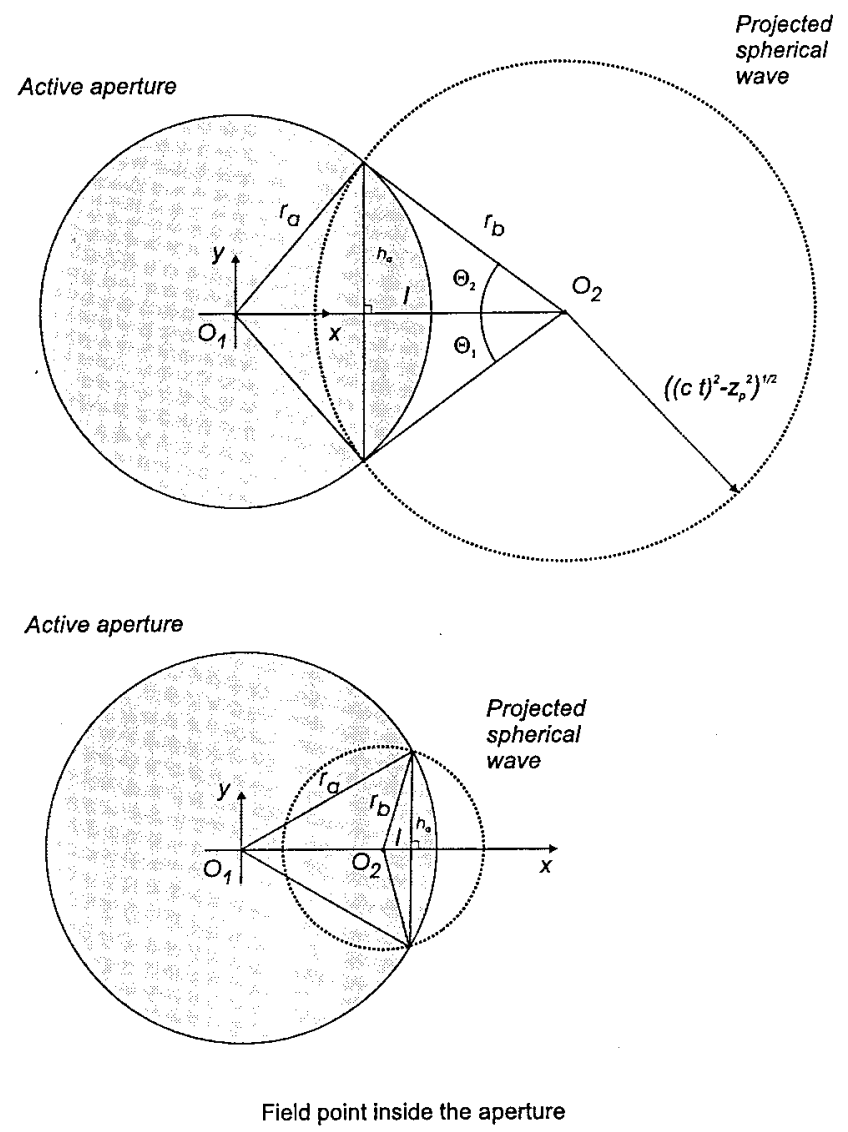

FIG. 7. Geometry for determining intersections between circles. The top graph shows the geometry when the field point denoted by $\mathrm{O}_{2}$ is outside the aperture, and the bottom graph when it is inside. 

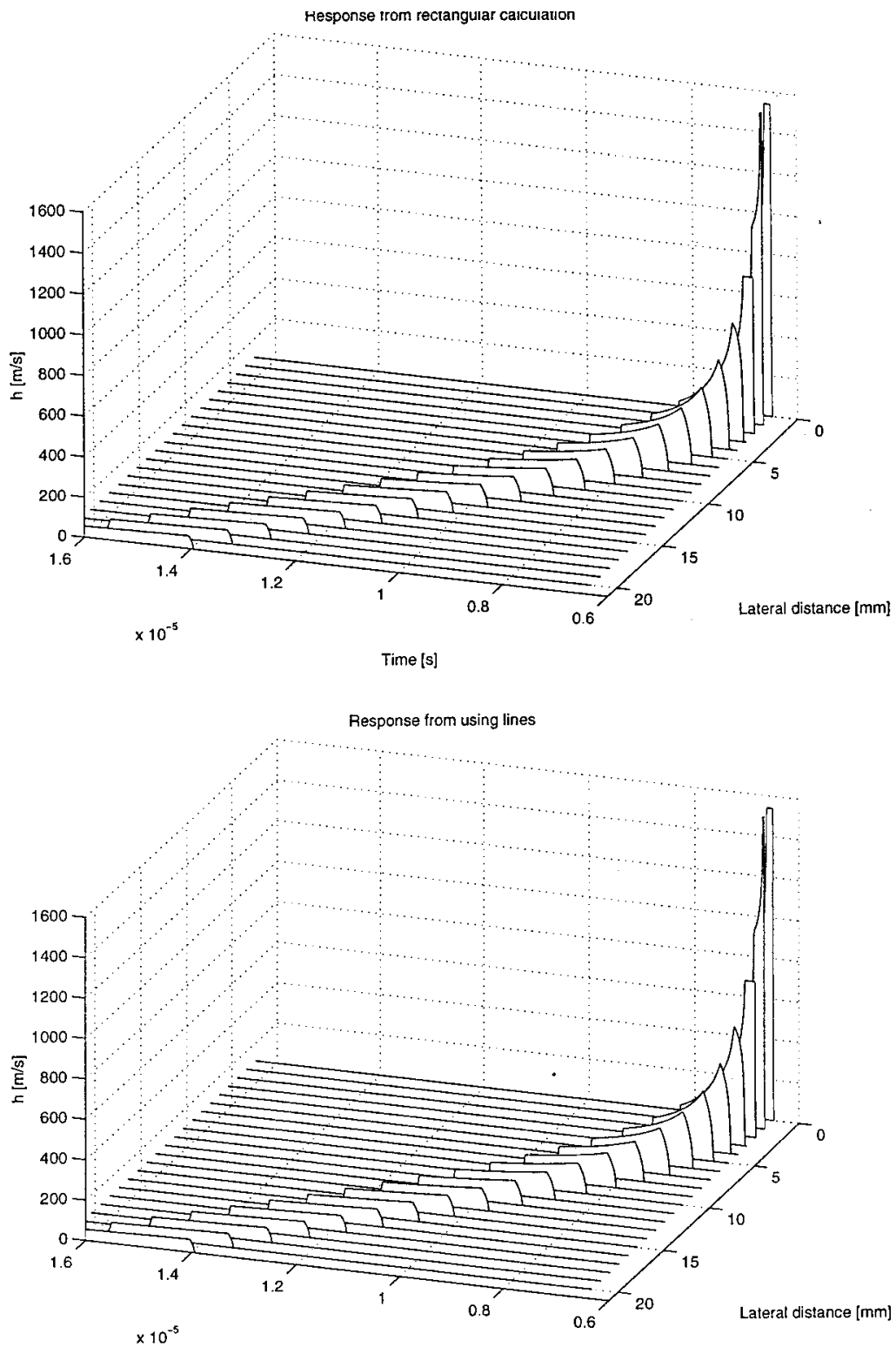

Time $[s$

and the projected spherical wave is centered at $O_{2}$ with radius $r_{b}(t)=\sqrt{(c t)^{2}-z_{p}^{2}}$. The length $h_{a}(t)$ is given by (Ref. 17 , p. 66)

$$
\begin{aligned}
& h_{a}(t)=\frac{2 \sqrt{p(t)(p(t)-a)\left(p(t)-r_{a}\right)\left(p(t)-r_{b}(t)\right)}}{a}, \\
& a=\left\|O_{1}-O_{2}\right\|, \\
& p(t)=\frac{a+r_{a}+r_{b}(t)}{2} .
\end{aligned}
$$

In a coordinate system centered at $O_{1}$ and an $x$-axis in the $O_{1}-O_{2}$ direction, the intersections are at

$$
\begin{aligned}
& y=h_{a}(t), \\
& l= \pm \sqrt{r_{b}^{2}(t)-h_{a}^{2}(t)} .
\end{aligned}
$$

The sign for $l$ depends on the position of the intersections. A negative sign is used if the intersections are for negative values of $x$, and positive sign is used for positive $x$ positions.
FIG. 8. Spatial impulse response calculated from a rectangular transducer of $4 \times 5 \mathrm{~mm}$. The top graph shows the result from using traditional evaluation and the bottom graph is when using the new method with four bounding lines. The axial distance to the field point is $10 \mathrm{~mm}$ and the response is calculated for lateral distances from 0 to $21 \mathrm{~mm}$ off-axis in steps of $1 \mathrm{~mm}$.
When the field point is outside the active aperture the spatial impulse response is

$$
\begin{aligned}
& h\left(\mathbf{r}_{1}, t\right)=\frac{\left|\Theta_{2}-\Theta_{1}\right|}{2 \pi} c=\frac{c}{\pi} \arctan \left(\frac{h_{a}(t)}{l}\right), \\
& \Theta_{2}=\arctan \left(\frac{h_{a}(t)}{l}\right)=-\Theta_{1} .
\end{aligned}
$$

It must be noted that a proper four-quadrant arctan should be used to give the correct response. An alternative formula is (Ref. 18, p. 19)

$$
\begin{aligned}
h\left(\mathbf{r}_{1}, t\right)= & \frac{c}{2 \pi} \arcsin \\
& \times\left(\frac{2 \sqrt{p(t)(p(t)-a)\left(p(t)-r_{a}\right)\left(p(t)-r_{b}(t)\right)}}{r_{b}^{2}(t)}\right), \\
= & \frac{c}{2 \pi} \arcsin \left(\frac{a h_{a}(t)}{r_{b}^{2}(t)}\right) .
\end{aligned}
$$

The start time $t_{s}$ for the response is found from 


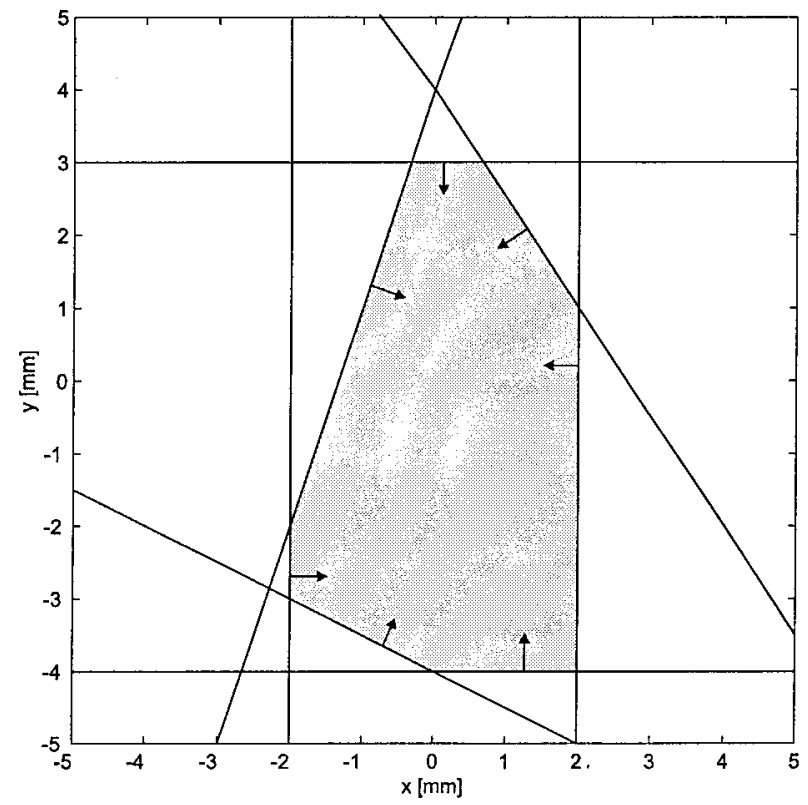

FIG. 9. Bounding lines defining a complex aperture. The arrows indicates the half-plane for the active aperture.

$$
\begin{aligned}
& r_{a}+r_{b}(t)=\left\|O_{1}-O_{2}\right\|, \\
& t_{s}=\frac{\sqrt{r_{b}^{2}(t)+z_{p}^{2}}}{c}=\frac{\sqrt{\left(\left\|O_{1}-O_{2}\right\|-r_{a}\right)^{2}+z_{p}^{2}}}{c},
\end{aligned}
$$

and the response ends at the time $t_{e}$ when

$$
\begin{aligned}
& r_{b}(t)=r_{a}+\left\|O_{1}-O_{2}\right\|, \\
& t_{e}=\frac{\sqrt{r_{b}^{2}(t)+z_{p}^{2}}}{c}=\frac{\sqrt{\left(\left\|O_{1}-O_{2}\right\|+r_{a}\right)^{2}+z_{p}^{2}}}{c} .
\end{aligned}
$$

When the field point is inside the aperture, the response is

$$
h\left(\mathbf{r}_{1}, t\right)=c \quad \text { for } \frac{Z_{p}}{c} \leqslant t \leqslant \frac{\sqrt{\left(r_{a}-\left\|O_{1}-O_{2}\right\|\right)^{2}+z_{p}^{2}}}{c} ;
$$

thereafter the arc lying outside the aperture should be subtracted, so that

$$
h\left(\mathbf{r}_{1}, t\right)=\frac{2 \pi-\left|\Theta_{2}-\Theta_{1}\right|}{2 \pi} c .
$$

The response ends when

$$
\begin{aligned}
& r_{b}(t)=r_{a}+\left\|O_{1}-O_{2}\right\|, \\
& t_{e}=\frac{\sqrt{\left(\left\|O_{1}-O_{2}\right\|+r_{a}\right)^{2}+z_{p}^{2}}}{c} .
\end{aligned}
$$

The determination of which part of the arc that subtracts or adds to the response is determined by what the active aperture is. One ring in an annular array can be defined as consisting of an active aperture outside a circle combined with an active aperture inside a circle for defining the inner and outer rim of the aperture. A circular aperture can also be combined with a line for defining the active area of a split aperture used for continuous wave probing.

\section{SOLUTION FOR PARAMETRIC SURFACES}

For ellipses or other higher-order parametric surfaces it is in general not easy to find analytic solutions for the spatial impulse response. The procedure described can, however, devise a simple solution to the problem, since the intersections between the projected spherical wave and the edge of the aperture uniquely determine the spatial impulse response. It is therefore possible to use root finding for a set of (nonlinear) equations for finding these intersections. The problem is to find when both the spherical wave and the aperture have crossing contours in the plane of the aperture, i.e., when

$$
\begin{aligned}
& (c t)^{2}-z_{p}^{2}-\left(x-x_{p}\right)^{2}-\left(y-y_{p}\right)^{2}=0, \\
& S(x, y)=0,
\end{aligned}
$$

in which $S(x, y)=0$ defines the boundary of the aperture. The problem of numerically finding these roots is in general not easy, if a good initial guess on the position of the inter-

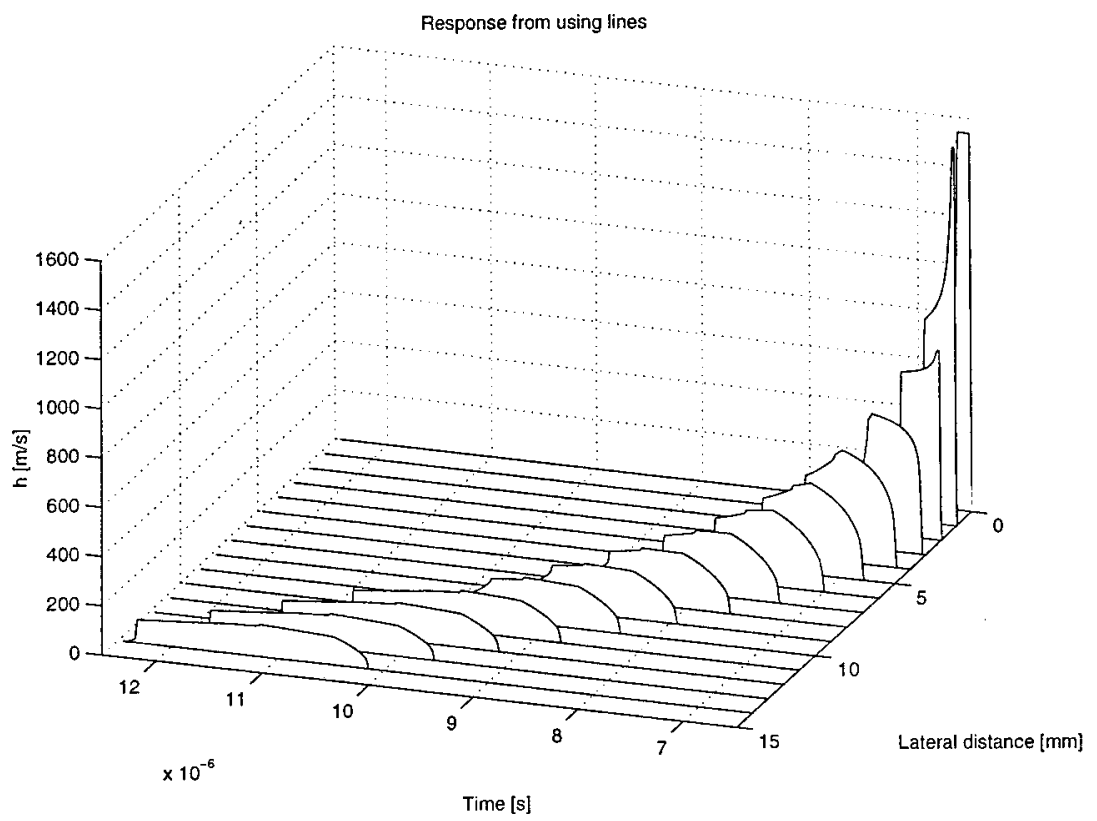

FIG. 10. Spatial impulse response from the complex aperture defined in Fig. 9. The axial distance to the field point is $10 \mathrm{~mm}$ and the response is calculated for lateral distances from 0 to $21 \mathrm{~mm}$ off-axis in the $x$-direction. 

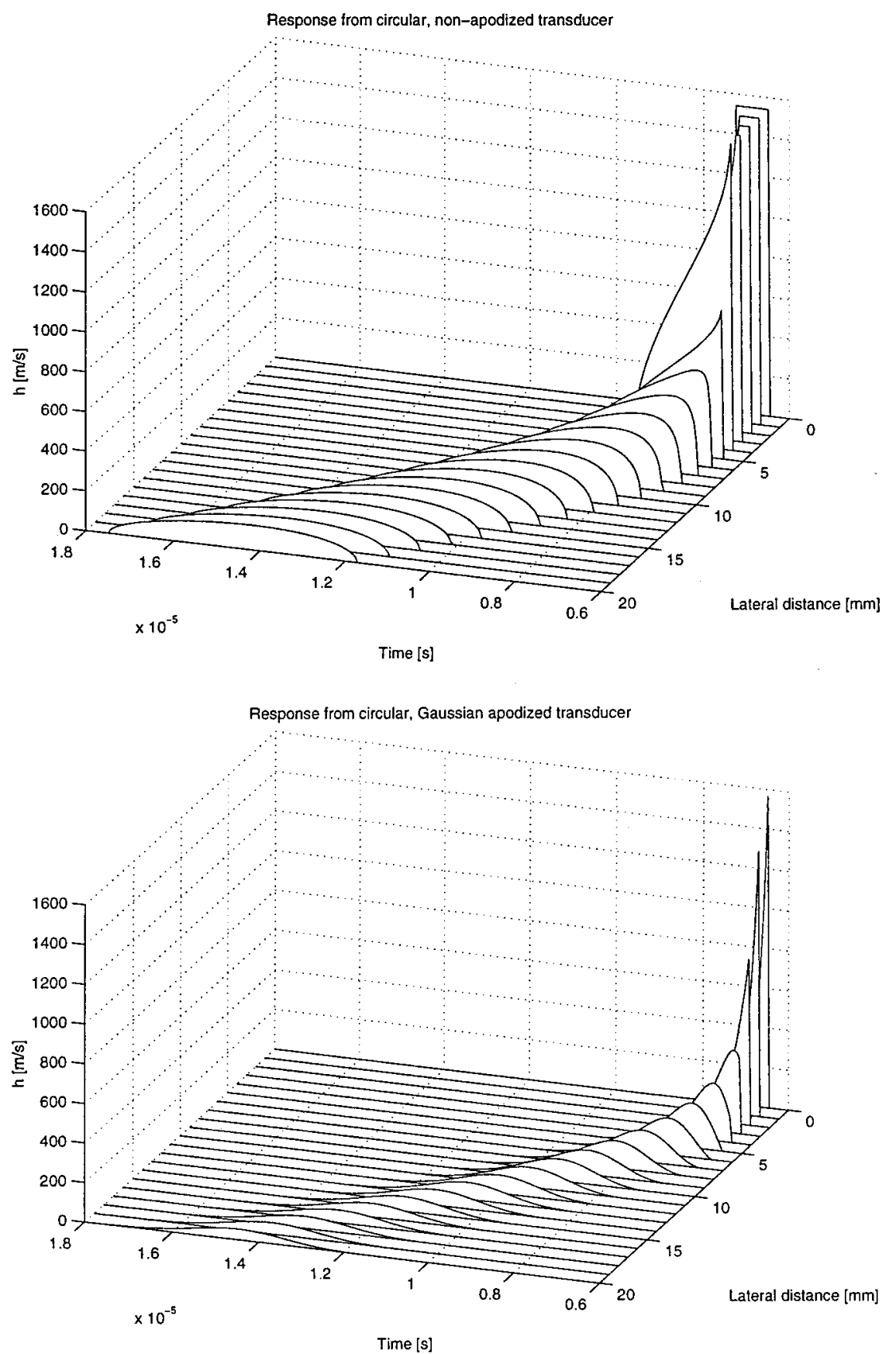

FIG. 11. Spatial impulse response from a circular aperture calculated with the new method. Graphs are shown without apodization of the aperture (top) and with a Gaussian apodization function (bottom). The radius of the aperture is $5 \mathrm{~mm}$ and the field is calculated $10 \mathrm{~mm}$ from the transducer surface. sections is not found (Ref. 19, pp. 286-289). Good initial values are, however, found here, since the intersections must lie on the projected circle and the intersections only move slightly from time point to time point. An efficient NewtonRaphson algorithm can therefore be devised for finding the intersections, and the procedure detailed here can be made to find the spatial impulse response for any flat transducer geometry with an arbitrary apodization and both hard and soft baffle mounting.

\section{EXAMPLES}

The first example shows a comparison between the traditional method for calculating spatial impulse responses and the new method. The response from a $4 \times 5 \mathrm{~mm}$ rectangle is found for different spatial positions $10 \mathrm{~mm}$ from the front face of the transducer. The responses are found from the center of the rectangle and out in steps of $1 \mathrm{~mm}$ in the $x$-direction to $21 \mathrm{~mm}$ away from the center of the rectangle. The results are shown in Fig. 8. It is seen that the two methods give identical results.

The second example is for a more complicated aperture, where its bounding lines are shown in Fig. 9. The calculated spatial impulse response is shown in Fig. 10. Responses have been calculated from the center position for $x=0 \mathrm{~mm}, y$ $=0 \mathrm{~mm}$ to the position $x=14 \mathrm{~mm}, y=0 \mathrm{~mm}$ in increments of $1 \mathrm{~mm}$. The distance to the transducer surface was always $10 \mathrm{~mm}(=z)$. A complicated response with a number of discontinuities is seen due to the many edges of the aperture.

The last example shows the response from a circular, flat transducer calculated with the new method. Two different cases are shown in Fig. 11. The top graph shows the tradi- 
tional spatial impulse response when no apodization is used, so that the aperture vibrates as a piston. The field is calculated $10 \mathrm{~mm}$ from the front face of the transducer starting at the center axis of the aperture. Twenty-one responses for lateral distance of $0-20 \mathrm{~mm}$ off-axis are then shown. The same calculation is repeated in the bottom graph, when a Gaussian apodization has been imposed on the aperture. The vibration amplitude is a factor of 1/exp(4) less at the edges of the aperture than at the center. It is seen how the apodization reduces some of the sharp discontinuities in the spatial impulse response.

\section{SUMMARY}

The general theory for the calculation of spatial impulse responses for flat transducer apertures has been reviewed. It was shown that the response can be found from the intersections of the projected spherical wave with the edges of the aperture. This made it possible to derive a general procedure for calculating these responses, which can be used for any flat geometry. It was also shown that the calculation easily can include hard and soft baffle mountings and the incorporation of any apodization function over the aperture. The approach makes it possible to make simulation programs that can handle arbitrary, flat, apodized aperture geometries without making approximations in the evaluation of the spatial impulse response for the hard baffle case.

${ }^{1}$ G. E. Tupholme, "Generation of acoustic pulses by baffled plane pistons," Mathematika 16, 209-224 (1969).

${ }^{2}$ P. R. Stepanishen, "Transient radiation from pistons in an infinte planar baffle," J. Acoust. Soc. Am. 49, 1629-1638 (1971).

${ }^{3} \mathrm{P}$. R. Stepanishen, "The time-dependent force and radiation impedance on a piston in a rigid infinite planar baffle," J. Acoust. Soc. Am. 49, 841-849 (1971).
${ }^{4}$ A. Penttinen and M. Luukkala, "The impulse response and nearfield of a curved ultrasonic radiator,'” J. Phys. D 9, 1547-1557 (1976).

${ }^{5}$ M. Arditi, F. S. Forster, and J. Hunt, “Transient fields of concave annular arrays," Ultrason. Imaging 3, 37-61 (1981).

${ }^{6}$ J. C. Lockwood and J. G. Willette, "High-speed method for computing the exact solution for the pressure variations in the nearfield of a baffled piston," J. Acoust. Soc. Am. 53, 735-741 (1973).

${ }^{7}$ J. L. S. Emeterio and L. G. Ullate, "Diffraction impulse response of rectangular transducers," J. Acoust. Soc. Am. 92, 651-662 (1992).

${ }^{8}$ J. A. Jensen, "Ultrasound fields from triangular apertures," J. Acoust. Soc. Am. 100, 2049-2056 (1996).

${ }^{9}$ J. A. Jensen and N. B. Svendsen, "Calculation of pressure fields from arbitrarily shaped, apodized, and excited ultrasound transducers," IEEE Trans. Ultrason. Ferroelectr. Freq. Control 39, 262-267 (1992).

${ }^{10} \mathrm{~J}$. A. Jensen, "Simulating arbitrary geometry ultrasound transducers using triangles," in Proc. IEEE Ultrason. Symp., Vol. 2 (IEEE, New York, 1996), pp. 885-888.

${ }^{11}$ G. R. Harris, "Review of transient field theory for a baffled planar piston," J. Acoust. Soc. Am. 70, 10-20 (1981).

${ }^{12}$ P. R. Stepanishen, "Wide bandwidth near and far field transients from baffled pistons," in Proc. IEEE Ultrason. Symp. (IEEE, New York, 1977), pp. $113-118$.

${ }^{13}$ G. R. Harris, "Transient field of a baffled planar piston having an arbitrary vibration amplitude distribution," J. Acoust. Soc. Am. 70, 186-204 (1981).

${ }^{14}$ P. R. Stepanishen, “Acoustic transients from planar axisymmetric vibrators using the impulse response approach," J. Acoust. Soc. Am. 70, 1176-1181 (1981).

${ }^{15}$ J. Naze Tjøtta and S. Tjøtta, "Near field and far field of pulsed acoustic radiators,"' J. Acoust. Soc. Am. 71, 824-834 (1982).

${ }^{16} \mathrm{~J}$. W. Goodman, Introduction to Fourier Optics, 2nd ed. (McGraw-Hill, New York, 1996).

${ }^{17}$ L. Raade and B. Westergreen, $\beta$ Mathematics Handbook (Charwell-Bratt, Ltd., Kent, England, 1990).

${ }^{18}$ M. R. Spiegel, Mathematical Handbook of Fornulas and Tables (McGraw-Hill, New York, 1968).

${ }^{19}$ W. H. Press, B. P. Flannery, S. A. Teukolsky, and W. T. Vetterling, Numerical Receipes in C. The Art of Scientific Computing (Cambridge University Press, Cambridge, 1988). 